\title{
Low doses of the mycotoxin citrinin protect cortical neurons against glutamate-induced excitotoxicity
}

\author{
Yui Nakajima*, Hirotoshi Iguchi*, Shinji Kamisuki, Fumio Sugawara, Teiichi Furuichi \\ and Yo Shinoda

\begin{abstract}
Department of Applied Biological Science, Faculty of Science and Technology, Tokyo University of Science, Noda, Chiba 278-8510, Japan
\end{abstract}

[Recommended by Toshiyuki Kaji]

(Received January 27, 2016; Accepted January 27, 2016)

\begin{abstract}
Citrinin, a natural mycotoxin that is found in fermented foods, is known as a cytotoxin and nephrotoxin. Exposure to high doses of citrinin result in apoptosis; however, the effects of low doses are not fully understood. Glutamate excitotoxicity is responsible for neuronal death in acute neurological disorders including stroke, trauma and other neurodegenerative diseases. Here, we show the neuroprotective effect of low doses of citrinin against glutamate-induced excitotoxicity. We examined the effect of citrinin exposure on glutamate-induced cell death in cultured rat cortical neurons under two conditions: simultaneous treatment with citrinin 0.1 to $1,000 \mathrm{nM}$ and glutamate $(30 \mu \mathrm{M})$ for $1,3 \mathrm{hr}$; the same simultaneous treatment for $3 \mathrm{hr}$ after pretreatment with citrinin for $21 \mathrm{hr}$. Both the MTT and immunocytochemical assay showed significant neuroprotective effects at several doses and exposure times tested. All concentrations of citrinin tested showed no remarkable cell death following 14-day exposure, and no marked alterations to synapses. These data suggest that low doses of citrinin can be used as a neuroprotective agent against glutamate-induced excitotoxicity without additional harmful cellular alterations.
\end{abstract}

Key words: Citrinin, Excitotoxicity, Glutamate, Cortical neuron

\section{INTRODUCTION}

Excitatory neurotoxicity in the mammalian central nervous system is largely mediated by excess amounts of glutamate (Choi, 1994; Sattler and Tymianski, 2001). Prolonged or unusual glutamate exposure induces neuronal cell death via strong calcium influx, resulting in acute neuronal injury such as stroke (Dirnagl et al., 1999), traumatic brain injury (Lee et al., 1999), hypoglycaemia (Facci et al., 1990) and epilepsy (Olney et al., 1986). It has been suggested that similar mechanisms contribute to the neuronal injury observed in neurodegenerative diseases such as amyotrophic lateral sclerosis (Shaw et al., 2001; Van Den Bosch et al., 2006), Alzheimer's disease (AD) (Schubert and Piasecki, 2001; Dong et al., 2009), Parkinson's disease (Blandini et al., 1996; Dong et al., 2009) and Huntington's disease (Estrada Sánchez et al., 2008; Dong et al., 2009).

Glutamate exposure in cultured neurons causes selective neuronal cell death that can be prevented by treat- ment with glutamate receptor antagonists (Michaels and Rothman, 1990; Albers et al., 1999; Arias et al., 1999), suggesting that glutamate excitotoxicity is induced by activation of glutamate receptors and their downstream machinery. Therefore, preventing these mechanisms appears to be one of the targets in drug discovery for glutamate excitotoxicity and related neurodegenerative disorders. Despite promising benefits for neuroprotective actions in vitro (Michaels and Rothman, 1990; Albers et al., 1999; Arias et al., 1999), the clinical use of glutamate receptor antagonists has not been strongly recommended (Muir and Lees, 1995) because of the psychotomimetic reactions observed in patients (Krystal et al., 1994; Grotta et al., 1995) and their ability to induce abnormal locomotor activities in rodents (Tricklebank et al., 1989; Löscher and Hönack, 1992). Memantine, a non-competitive NMDA receptor antagonist, has already been used as a neuroprotective drug against glutamate excitotoxicity (Mount and Downton, 2006) for patients with AD; however, memantine only shows small beneficial effects

Correspondence: Teiichi Furuichi (E-mail: tfuruichi@rs.tus.ac.jp)

Yo Shinoda (E-mail: yshinoda-ns@umin.net)

*These authors equally contributed to this work. 
for patients suffering from moderate-to severe AD and does not show any effect for mild AD patients (Schneider et al., 2011). Furthermore, memantine potentially affects basal synaptic transmission so that some neurologic symptoms are induced by memantine treatment (Villoslada et al., 2009). Hence, development of neuroprotective drugs against glutamate excitotoxicity, which are not glutamate receptor antagonists, is required.

Citrinin is known as a nephrotoxic mycotoxin produced by several fungal strains belonging to Penicillium, Aspergillus and Monascus (Flajs and Peraica, 2009). Citrinin has also been identified as a contaminant in various types of human foods such as corn, wheat, rice, barley, maize, rye, oats and nuts (Scott et al., 1972; Nelson et al., 1985; Abramson et al., 2001). The toxicities of citrinin have been frequently reported as cytotoxic (50-220 $\mu \mathrm{M}, 24-72 \mathrm{hr}$ as $\mathrm{LC}_{50}$, which depends on cell type) (Liu et al., 2003; Yu et al., 2006; Bouslimi et al., 2008) and nephrotoxic via swelling of the kidney and acute tubular necrosis $\left(50-134 \mathrm{mg} / \mathrm{kg}\right.$ orally as $\mathrm{LD}_{50}$, which depends on species) (Ambrose and DeEds, 1946; Friis et al., 1969; Krogh et al., 1970). The mechanism of citrinin toxicity is thought to involve mitochondrial dysfunction (Aleo et al., 1991; Chagas et al., 1992a, 1992b; Da Lozzo et al., 1998). Furthermore, citrinin affects intracellular calcium level (Berndt et al., 1984; Chan, 2008; Klarić et al., 2012) so that citrinin has the potential to influence the cellular events regulated by calcium influx. These effects were observed at high doses $(\sim 100 \mu \mathrm{M})$; however, the effect of low doses remains unclear.

In the present study, we investigated the effect of the citrinin on glutamate-induced excitotoxicity in rat cortical neuronal cultures. We demonstrate that exposure to low doses of citrinin showed a significant neuroprotective effect against glutamate excitotoxicity. Importantly, low doses of citrinin did not induce any cell death after 2 weeks of exposure. Furthermore, low doses of citrinin exposure did not alter synapse status. These results suggest that low doses of citrinin could potentially be used as a neuroprotective drug against glutamate-induced excitotoxicity.

\section{MATERIALS AND METHODS}

\section{Cortical cell culture}

All experimental protocols were evaluated and approved by the Regulation for Animal Research at Tokyo University of Science, and were carried out in accordance with the approved protocols. Cortical neurons from Wister rats were prepared as described previously (Shinoda et al., 2010) with partial modifications. At embryonic day 18 , the pregnant rat was deeply anaes- thetised, the pups were decapitated and the brains were removed into ice-cold HEPES-buffered salt solution (Life Technologies, Tokyo, Japan). Cortical tissue was digested using papain (Sigma-Aldrich, Tokyo, Japan), triturated, and plated on poly-ethylene imine (Sigma-Aldrich) coated coverslips and/or 96-well plates, and cultured in Neurobasal Medium (Life Technologies) supplemented with B-27 (Life Technologies) and GlutaMAX (Life Technologies). Cultures were maintained at $37^{\circ} \mathrm{C}$ in a humidified atmosphere of $5 \% \mathrm{CO}_{2}$ and $95 \%$ air. At 14 and 15 days in vitro (DIV), cultured cells were treated with citrinin (dissolved to DMSO, vehicle was added for control) and glutamate.

\section{Drug treatment and MTT assay}

Citrinin (extracted from the culture broth of a fungus by Kamisuki and Sugawara) was applied to neurons at 14 (for $24 \mathrm{hr}$ treatment) and 15 (for 1 and $3 \mathrm{hr}$ treatment) DIV. Glutamate $(30 \mu \mathrm{M})$ was added to citrinintreated cells for $3 \mathrm{hr}$ as schematized in Fig. 1A. The MTT assay was performed using the MTT cell growth assay kit (Millipore, Billerica, MA, USA). MTT (5 mg/mL) stock solution dissolved in PBS was added directly to the culture medium at a final concentration of 50 $\mu \mathrm{g} / \mathrm{mL}$ and incubated at $37^{\circ} \mathrm{C}$ in a humidified atmosphere of $5 \% \mathrm{CO}_{2}$ and $95 \%$ air for $1 \mathrm{hr}$. The medium was completely removed and $100 \mu \mathrm{L}$ dimethyl sulfoxide was added to dissolve the formazan product. To calculate cell viability, the absorbance was measured with a test wavelength at $570 \mathrm{~nm}$ and a reference wavelength at $630 \mathrm{~nm}$ using a microplate reader (Molecular Devices, Sunnyvale, CA, USA), and subtracted the $630 \mathrm{~nm}$ background absorbance from the $570 \mathrm{~nm}$ measurement. For long-term viability assays, citrinin was applied to neurons at 14 DIV for 1, 2, 5 and 14 days. The MTT assay was performed as described above. Cell viability is presented as the mean \pm S.E.M. from three independent cultures. Statistical comparisons were performed using one-way analysis of variance (ANOVA) and the Tukey-Kramer post hoc test.

\section{Immunocytochemistry}

Immunocytochemistry was performed as previously described (Shinoda et al., 2011; Shinoda et al., 2014) with slight modification. Cells were fixed with $4 \%(\mathrm{w} / \mathrm{v})$ PFA/0.1 M phosphate buffer and then washed with PBS. After blocking with $2 \%(\mathrm{v} / \mathrm{v})$ normal donkey serum, $0.1 \%$ Triton and $0.05 \% \mathrm{NaN}_{3}$ in $2 \mathrm{x} \mathrm{PBS}$, cells were incubated with primary antibodies at room temperature for $1 \mathrm{hr}$ or $4^{\circ} \mathrm{C}$ overnight, rinsed with $\mathrm{PBS}$, and incubated with secondary antibodies at room temperature for $1 \mathrm{hr}$. After washing with PBS, cells were mounted with Fluoromount/ 
Citrinin protect neurons against glutamate-induced excitotoxicity

(A)

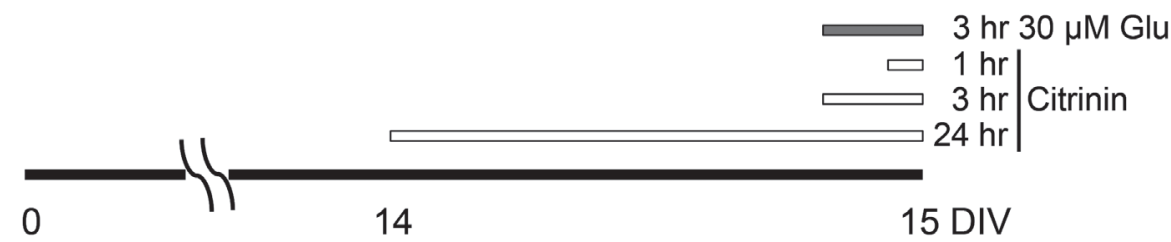

(B)
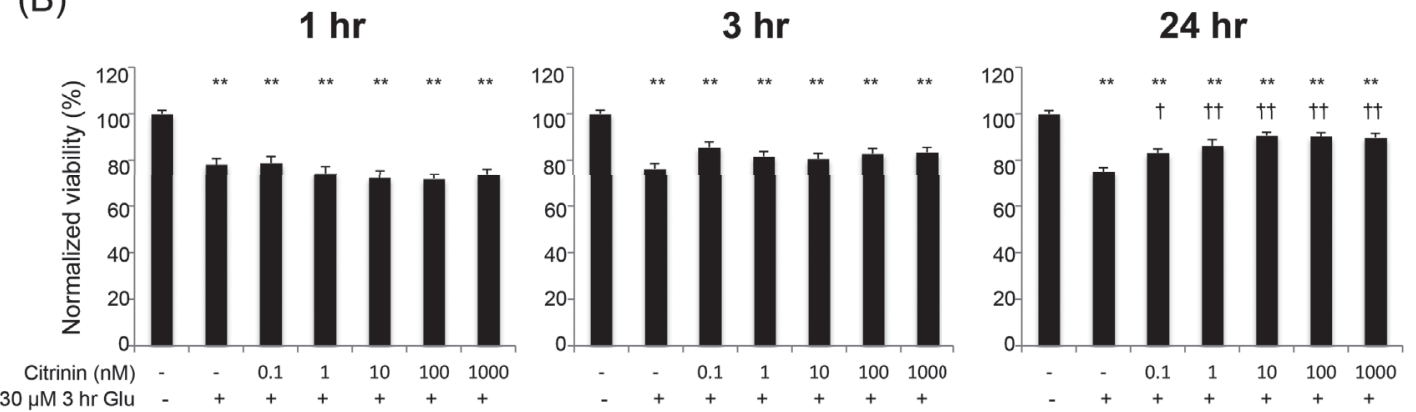

Fig. 1. Low-dose exposure to citrinin is neuroprotective against glutamate-induced excitotoxicity, as revealed by the MTT assay. (A) Schematic diagram of short- and long-term citrinin treatment and glutamate stimulation. (B) Cell viability and the effect of different concentrations of citrinin. Cells were exposed to each dose of citrinin and times together with $30 \mu \mathrm{M}$ glutamate for $3 \mathrm{hr}$. The data represent the mean \pm S.E.M. $(\mathrm{n}=24) . * * P<0.01$ compared with no drug- and glutamate-treated culture. $\dagger \dagger P<0.01$ and $\dagger P<0.05$ compared with $30 \mu \mathrm{M}$ glutamate-treated culture. One-way ANOVA and post hoc Tukey-Kramer test.

Plus (Diagnostic BioSystems, Pleasanton, CA, USA). Fluorescent imaging was performed using the epifluorescent microscope Eclipse E800 (Nikon, Tokyo, Japan) equipped with a cooled CCD camera SPOT (Diagnostic Instruments), or epifluorescent microscope Eclipse TE2000-E (Nikon) equipped with a CoolSNAP HQ2 CCD camera (Photometrics, Tucson, AZ, USA). The ratio of living neuron $(\mathrm{MAP} 2 / \mathrm{NeuN})$ is presented as the mean \pm S.E.M. from three independent experiments. All neurons were counted by NeuN immunopositive nuclei using ImageJ (Wayne Rasband, Maryland, DC, USA), and living neurons were identified and counted by MAP2 staining with healthy neurites. The numbers of excitatory and inhibitory terminals were counted along $20 \mu \mathrm{m}$ length dendrites. The numbers of each terminal are presented as the mean \pm S.E.M. from four independent experiments. Statistical comparisons were performed using one-way ANOVA and the Tukey-Kramer post hoc test. The following primary antibodies were used: mouse anti-NeuN (Millipore), chicken anti-MAP2 (Millipore), mouse anti-vGluT1 (Synaptic Systems, Göttingen, Germany) and rabbit anti-vGAT (Synaptic Systems) antibody. All Alexa dye conjugated secondary antibodies were obtained from Life Technologies.

\section{Calcium imaging}

Calcium influx in cortical neurons was observed by ratiometric imaging of the $\mathrm{Ca}^{2+}$-sensitive dye Fura-2 AM (Nacalai Tesque, Kyoto, Japan). Briefly, 15 DIV drugtreated cortical neurons were loaded with $2 \mu \mathrm{M}$ Fura-2 $\mathrm{AM}$ for at least $40 \mathrm{~min}$ at $37^{\circ} \mathrm{C}, 5 \% \mathrm{CO}_{2}$. Cells were then washed in HEPES-buffered solution (HBS; $150 \mathrm{mM}$ $\mathrm{NaCl}, 4 \mathrm{mM} \mathrm{KCl}, 2 \mathrm{mM} \mathrm{MgCl}, 2 \mathrm{mM} \mathrm{CaCl}_{2}, 10 \mathrm{mM}$ glucose, $10 \mathrm{mM}$ HEPES, pH 7.3) to remove excess Fura-2 $\mathrm{AM}$ and incubated in HBS for another $30 \mathrm{~min}$ at $37^{\circ} \mathrm{C}$ to equilibrate. The coverslips with cells loaded with Fura-2 AM was then mounted in a recording chamber (Warner Instruments, Greenwich, CT, USA) containing $100 \mu \mathrm{L}$ HBS and imaged using an inverted microscope Ti-U (Nikon). Baseline was obtained $30 \mathrm{sec}$ prior to the application of glutamate. Glutamate stimulation was performed by application of additional $100 \mu \mathrm{L}$ HBS containing $60 \mu \mathrm{M}$ glutamate to achieve a concentration of $30 \mu \mathrm{M}$ glutamate. Fura-2 was excited using a mercury light source at $340 \mathrm{~nm}$ and $380 \mathrm{~nm}$ by means of two narrow beam band pass filters (340/26 and 387/11) selected by a computer-controlled filter wheel. The emitted fluorescence was filtered through a 510/84 filter, captured with an EMCCD camera iXon (Andor Technology, Tokyo, Japan), and analysed with NIS-Elements software (Nikon). The fluorescent ratio were calculated and 
normalised to the baseline. Changes in calcium influx are presented as the mean \pm S.E.M. from three or more independent experiments with $\geq 8$ cells per experiment. Statistical analysis was performed using the Student's $t$-test.

\section{RESULTS}

\section{Exposure to low doses of citrinin are neuroprotective against glutamate excitotoxicity}

To examine the effect of low-dose citrinin treatment on glutamate-induced excitotoxicity, we compared the cell viability of rat cortical neurons following short- $(1 \mathrm{hr}$ or $3 \mathrm{hr}$ ) and long-term (24 hr) citrinin treatment after $30 \mu \mathrm{M}$ glutamate exposure for $3 \mathrm{hr}$ using the MTT assay (Fig. 1A). In the case of short-term treatment, cells were treated with glutamate $2 \mathrm{hr}$ before citrinin application $(1 \mathrm{hr})$ or simultaneously treated with citrinin and glutamate for $3 \mathrm{hr}(3 \mathrm{hr})$. In the case of long-term treatment, cells were pretreated with citrinin for $21 \mathrm{hr}$ prior to simultaneous treatment for $3 \mathrm{hr}$ with glutamate ( $24 \mathrm{hr}$ ). Glutamate exposure induced significant cell death (the viability is $78.2 \pm 2.6,76.4 \pm 2.2$ and $75.1 \pm 1.8 \%$ control in 1,3 and $24 \mathrm{hr}$, respectively, $P<0.01)$. Short term ( $1 \mathrm{hr}$ and $3 \mathrm{hr}$ ) application of citrinin showed no neuroprotective effect against glutamate excitotoxicity $(78.9 \pm 2.8,74.4 \pm 2.9$, $72.2 \pm 3.2,71.8 \pm 2.0$ and $73.8 \pm 2.3 \%$ control for $1 \mathrm{hr}$; $85.6 \pm 2.4,81.8 \pm 1.9,80.6 \pm 2.4,82.9 \pm 2.1$ and $83.5 \pm$ $2.1 \%$ control for $3 \mathrm{hr}$; for $0.1,1,10,100$ and 1,000 nM, respectively), however, the significant neuroprotective effect was seen in long-term (24 hr) citrinin treatment at all citrinin doses $(83.1 \pm 1.7,86.4 \pm 2.6,90.8 \pm 1.3,90.2$ \pm 1.7 and $90.0 \pm 1.7 \%$ control for $0.1,1,10,100$ and 1000 nM, respectively, $P<0.01$; Fig. 1B).

Although the MTT assay is useful for the biochemical assessment of living cells by analysing mitochondrial

(A)
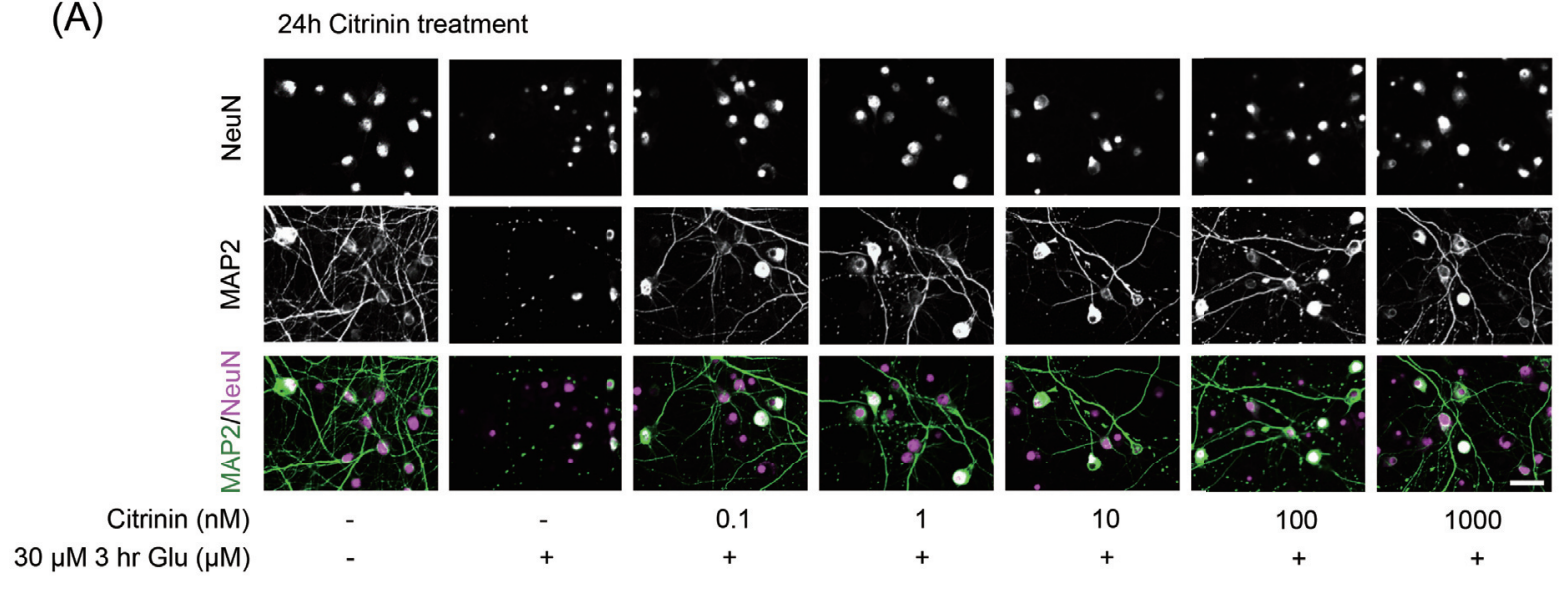

0.1

10

100

1000

(B)
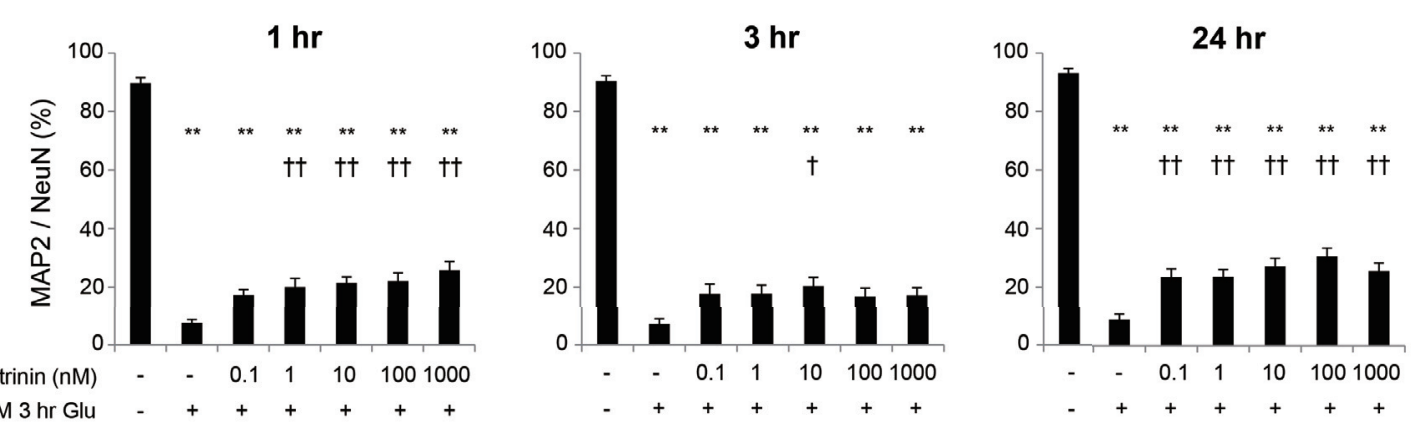

Fig. 2. Low-dose exposure to citrinin is neuroprotective against glutamate-induced excitotoxicity, as revealed by the nuclei staining assay. (A) Representative pictures of staining by NeuN (magenta) and MAP2 (green) after $24 \mathrm{hr}$ citrinin treatment in each doses with glutamate. Scale bar $=30 \mu \mathrm{m}$. (B) Summary of 1,3 and $24 \mathrm{hr}$ citrinin treatment with $3 \mathrm{hr}$ glutamate exposure. Data represent the mean \pm S.E.M. $(1 \mathrm{hr}: \mathrm{n}=23,24,24,24,24,24,24 ; 3 \mathrm{hr}: \mathrm{n}=30,28,30,30,29,29,30 ; 24 \mathrm{hr}: \mathrm{n}=18,18,18,18,17,17,17$ from left to right). $* * P<0.01$ compared with no drug and glutamate-treated cultures. $\uparrow \uparrow P<0.01$ and $\uparrow P<0.05$ compared with $30 \mu \mathrm{M}$ glutamate-treated cultures. One-way ANOVA and post hoc Tukey-Kramer test. 
Citrinin protect neurons against glutamate-induced excitotoxicity

activity, this assay does not distinguish among cell types such as neurons and glia that are always co-cultured in primarily culture. Therefore, we further confirmed the neuroprotective effect of citrinin against glutamate excitotoxicity using immunocytochemistry (Fig. 2). NeuN immunopositive staining was used as neuronal marker, and MAP2 staining and their stained neurites was used as the marker of healthy neuron. Therefore, the ratio of the healthy neuron can be calculated by divided the number of healthy MAP2 immunopositive neurons by the number of all NeuN positive neurons. In this experiment, glutamate exposure showed significant neuronal death comparing with control (Fig. 2B: the viability is $89.7 \pm 1.8$, $90.5 \pm 1.8$ and $93.1 \pm 1.6 \%$ for control; $7.5 \pm 1.1,7.0 \pm$ 1.8 and $8.9 \pm 2.0 \%$ for $30 \mu \mathrm{M}$ glutamate in $1,3,24 \mathrm{hr}$, respectively, $P<0.01)$. Unlike the results of MTT assay, short-term citrinin treatment shows significant neuroprotective effect compared to glutamate exposure group (Fig. 2B: $17.2 \pm 2.0,20.0 \pm 2.9,21.4 \pm 2.1,22.0 \pm 2.9$ and $25.7 \pm 2.9 \%$ for $1 \mathrm{hr} ; 17.6 \pm 3.4,17.7 \pm 3.0,20.3 \pm$ $3.0,16.8 \pm 2.8$ and $17.1 \pm 2.7 \%$ for $3 \mathrm{hr} ; 0.1,1,10,100$ and $1,000 \mathrm{nM}$, respectively, $P<0.01$ and 0.05$)$ as well as long-term citrinin treatment group $(23.4 \pm 2.9,23.5 \pm 2.5$, $27.1 \pm 2.8,30.5 \pm 2.9$ and $25.5 \pm 2.8 \% ; 0.1,1,10,100$ and $1,000 \mathrm{nM}$ for $24 \mathrm{hr}$, respectively, $P<0.01)$. These data suggest that low dose citrinin has neuroprotective effect against glutamate induced excitotoxicity.

\section{Long-term exposure to citrinin does not induce neuronal cell death}

To evaluate citrinin as a neuroprotective candidate compound, we tested if citrinin caused neuronal damage during extended periods of treatment. Cultures were treated with citrinin for up to 14 days and were analysed using the MTT assay (Fig. 3). Relatively low doses (0.1-10 $\mu \mathrm{M})$ of citrinin did not induce significant cell death compared with control cultures; however, high doses $(100 \mu \mathrm{M})$ of citrinin showed significant cell death at all timepoints tested $(74.8 \pm 2.5,59.2 \pm 1.8,18.3 \pm 1.1$ and 1.5 $\pm 0.1 \%$ for $1-, 2-, 5$ - and 14-day exposure, respectively, $* * * P<0.001, * * P<0.01)$. These results suggest that exposure to low doses of citrinin for at least 14 days does not induce cell death.

\section{Exposure to low doses of citrinin does not alter synapse status}

In addition to cell viability, subcellular effects such as alterations to synaptic connectivity were also analysed. To verify whether citrinin affects synaptic status, we analysed the number of excitatory and inhibitory synapses along dendrites in citrinin-treated neurons (Fig. 4). Exci-

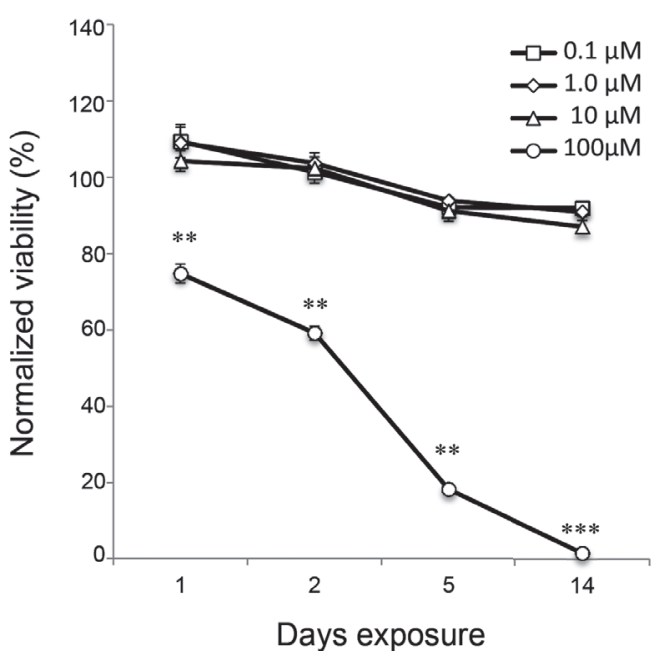

Fig. 3. Long-term low-dose exposure to citrinin does not induce cell death. Cell viability after 14 day exposure to each dose of citrinin normalised to no citrinin-treated cultures. Exposure to $0.1-10 \mu \mathrm{M}$ of citrinin did not induce significant cell death for 14 days. Data represent the mean \pm S.E.M. ( $\mathrm{n}=9$ for all group). $* * P<0.01$ One-way ANOVA and post hoc Tukey-Kramer test.

tatory and inhibitory synaptic terminals were visualised using the synaptic marker antibodies vGluT1 and vGAT, respectively, and the neuronal dendrite marker MAP2 (Fig. 4A). Both excitatory (Fig. 4B, 14.1 $\pm 0.7,13.7 \pm$ $1.0,13.5 \pm 0.9$ and $14.0 \pm 0.8$ from 0 to $10 \mu \mathrm{M}$ citrinin treatment, respectively) and inhibitory (Fig. 4C, $3.7 \pm 0.7$, $3.7 \pm 0.5,3.7 \pm 0.5$ and $3.5 \pm 0.4$ from 0 to $10 \mu \mathrm{M}$ citrinin treatment, respectively) synaptic terminals remained unaltered when cells were treated with low doses of citrinin. These data suggest that citrinin treatment did not affect neuronal synapse status.

\section{Low-dose exposure to citrinin does not change glutamate induced $\mathrm{Ca}^{2+}$ influx}

One of the most important triggers of glutamate excitotoxicity is intracellular calcium overload (Szydlowska and Tymianski, 2010). To clarify whether the neuroprotective effect of citrinin is attributable to the inhibition of calcium overload, calcium imaging experiments were performed. Calcium influx was measured by ratiometric fluorescent imaging with the calcium indicator Fura-2. Application of $30 \mu \mathrm{M}$ glutamate to $0.1 \mu \mathrm{M}$ citrinin-treated (24 hr) neurons induced an increase in cytosolic calcium concentration (Fig. 5). The initial calcium influx was rapid and a subsequent elevation of intracellular calcium concentration was observed. Compared with control neurons, there was no significant difference between control 


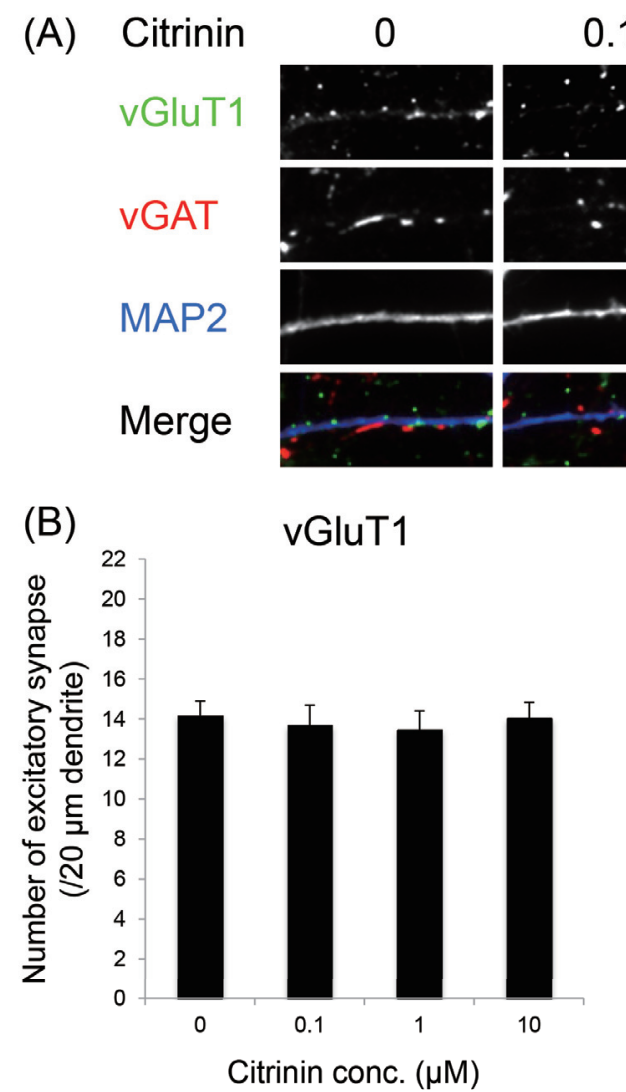

0.1

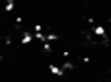

$\because$

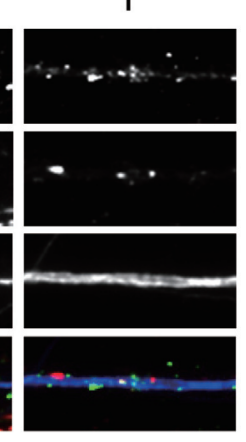

1 $10(\mu \mathrm{M})$
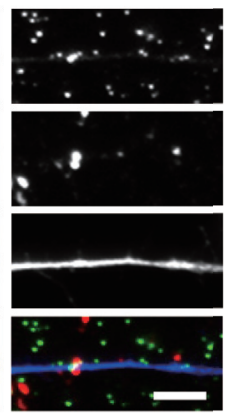

(C)

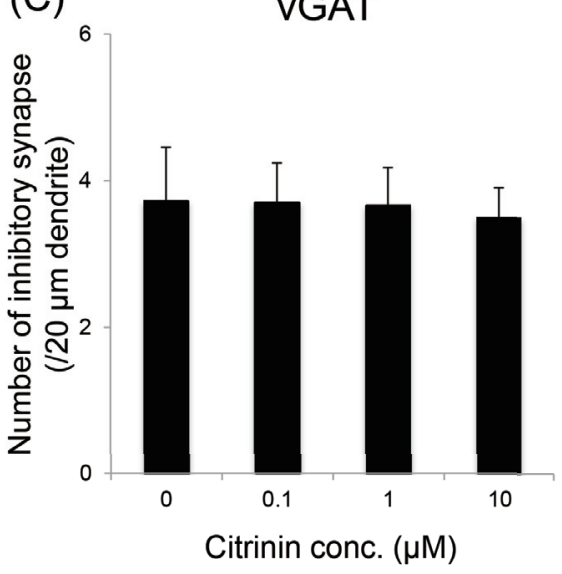

Fig. 4. Low-dose exposure to citrinin does not alter synapse status. (A) Representative pictures of fluorescent immunostaining of synapses 2 days after citrinin treatment. Excitatory (vGluT1) and inhibitory (vGAT) synapses along secondary dendrites (MAP2) were counted. Scale bar $=5 \mu \mathrm{m}$. (B) The number of vGluT1-immunopositive excitatory synapses along $20 \mu \mathrm{m}$ dendrites. Data represent the mean \pm S.E.M. ( $\mathrm{n}=67,36,43,38$ from left to right). One-way ANOVA. (C) The number of vGAT-immunopositive inhibitory synapses along $20 \mu \mathrm{m}$ dendrites. Data represent the mean \pm S.E.M. $(\mathrm{n}=11,17,15,14$ from left to right). One-way ANOVA.

and citrinin-treated neurons in both initial and subsequent increments of cytosolic calcium $(2.1 \pm 0.2,2.3 \pm 0.2$ for control and $0.1 \mu \mathrm{M}$ citrinin at $60 \mathrm{sec}$ after glutamate administration, respectively). These data suggest that the neuroprotective effect of citrinin is not directly related to the mechanism of calcium influx.

\section{DISCUSSION}

Here, we report for the first time, that exposure to low doses of citrinin are able to inhibit glutamate excitotoxicity and subsequent neuronal death. There are dozens of reports showing that high doses of citrinin act as a cytotoxin and nephrotoxin (Ambrose and DeEds, 1946; Friis et al., 1969; Krogh et al., 1970; Scott et al., 1972; Nelson et al., 1985; Aleo et al., 1991; Chagas et al., 1992a, 1992b; Da Lozzo et al., 1998; Abramson et al., 2001; Liu et al.,
2003; Yu et al., 2006; Bouslimi et al., 2008). In contrast, our results indicate a new role/implication for low-dose citrinin in health and disease. In the present study, we used glutamate excitotoxicity as a cellular model to mimic the penumbra in focal ischemic stroke, and studied the effect of citrinin on neuronal death in cultured rat cortical neurons. Our main findings of this study are that lowdose citrinin treatment (1) prevents glutamate excitotoxicity and subsequent neuronal death, (2) does not induce cell death during extended periods of exposure for at least 14 days, (3) does not alter the number of excitatory and inhibitory terminals, and (4) does not affect glutamate-induced calcium influx. These results indicate that low-dose citrinin is a potent lead compound candidate for neuroprotection against glutamate excitotoxic neurodegeneration and related neuronal diseases.

Previous studies have shown that citrinin acts as a 
Citrinin protect neurons against glutamate-induced excitotoxicity

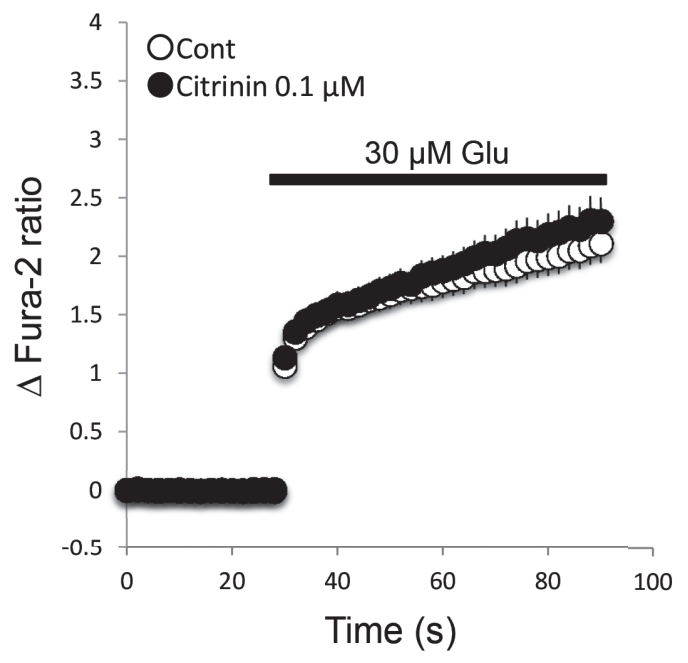

Fig. 5. Low-dose exposure to citrinin does not alter intracellular levels of $\mathrm{Ca}^{2+}$. Calcium response to glutamate exposure in $24 \mathrm{hr}$ citrinin-treated neurons. Data represent the mean \pm S.E.M. ( $\mathrm{n}=39$ for control, $\mathrm{n}=25$ for citrinin). Fura-2 ratio did not show any significant change between the control- and citrinin-treated groups immediately or $60 \mathrm{sec}$ after glutamate application. Student's $t$-test.

nephrotoxin, embryocidal toxin, fetotoxin and genotoxin (Flajs and Peraica, 2009) [for review]. The toxic effects of citrinin observed in vitro were reported in HEK 293 cells (Liu et al., 2005), mouse embryonic stem cells (Chan, 2008), CHO-K1 cells (Liu et al., 2003), and V79 cells (Pfeiffer et al., 1998). These studies used 10-100 $\mu \mathrm{M}$ citrinin to assess the toxic effects, with lower concentrations having no significant effect (Pfeiffer et al., 1998). In the present study, we used $0.1-1,000 \mathrm{nM}$ citrinin to evaluate its neuroprotective effect against glutamate excitotoxicity. Even though there are no significant neuroprotective effect for $1 \mathrm{hr}$ and $3 \mathrm{hr}$ citrinin treatment revealed by MTT assay, immunocytochemical study showed that significant effect in these short-term treatments. This contradiction could be explained by the relatively small neuroprotective effect in the short-term citrinin treatment. Small neuroprotective effect is thought to be difficult to determine by MTT assay. These concentrations did not induce any neuronal death or synaptic alterations. Taken together, our data suggest that low doses of citrinin have the potential to prevent glutamate excitotoxicity without any obvious side effects regarding neuronal cell death and synapse status. If citrinin is to be used as a neuroprotective drug, it must cross the blood-brain barrier (BBB). Although the direct measurement of citrinin concentration in the brain and estimation of BBB permeability is required, it has been reported that intraperitoneal admin- istration of citrinin alters some enzymes and metabolites in the brain (Gupta et al., 1986). In spite of the secondary effect of citrinin, such as alteration to the metabolism of endothelial cells, this report indicates that citrinin may have the ability to penetrate the BBB and affect neuronal metabolism.

The mechanisms of glutamate excitotoxicity have been widely investigated (Lau and Tymianski, 2010) [for review]. The detailed mechanism is not well understood; however, some possible hypotheses have been proposed (Dong et al., 2009; Lau and Tymianski, 2010; Wang and Qin, 2010). The important hypothetical steps of glutamate excitotoxicity are: (1) activation of ionotropic and metabotropic glutamate receptors, and subsequent membrane depolarisation; (2) excess ion influx, including calcium via membrane channels and/or ER localised channels; (3) oxidative stress elicited by aberrant production of reactive oxygen species (ROS); and (4) mitochondrial dysfunction. In the present study, we investigated one of the most important and primary steps of the glutamate excitotoxicity cascade, calcium influx. Interestingly, citrinin treatment did not alter calcium influx. Therefore, the neuroprotective effect of citrinin against glutamate induced excitotoxicity can not be explained by the prevention of calcium overload. There have been repeated reports that high-dose citrinin application induces the production of ROS and subsequent cell death (Chen and Chan, 2009; Kumar et al., 2011; Máté et al., 2014). Conversely, citrinin and its predicted precursor and metabolites have been reported as antioxidants (Li et al., 2006; Zhang et al., 2007). In order to understand these properties of citrinin, the low-dose effect of citrinin on ROS production or inhibition should be further investigated.

In conclusion, the present study reveals for the first time that low-dose citrinin treatment prevents glutamateinduced neuronal excitotoxicity and subsequent cell death without changing synapse status. The detailed mechanism of the neuroprotective effect of citrinin is still unknown. However, citrinin treatment does not affect glutamate-induced calcium influx, further investigations are required to clarify the mechanism. In addition, in vivo experiments confirming the effects of citrinin are required for effective drug discovery against glutamate excitotoxicity and related neurodegenerative disorders.

\section{ACKNOWLEDGMENTS}

Financial support was provided by KAKENHI from the Japan Society for the Promotion of Science (JSPS) in the Ministry of Education, Culture, Sports, Science and Technology of Japan (MEXT) and the Grant in Aid for 
Neuroscience Research from the Narishige Neuroscience Research Foundation. The authors declare no competing financial interests.

\section{Author contributions}

Y.S. conceived the experiments. Y.N., H.I. and Y.S. conducted the experiments. S.K., F.S. extracted citrinin. Y.N., H.I., T.F. and Y.S. analysed the results. Y.N., H.I. and Y.S. wrote the paper. All authors reviewed the manuscript.

Conflict of interest---- The authors declare that there is no conflict of interest.

\section{REFERENCES}

Abramson, D., Usleber, E. and Märtlbauer, E. (2001): Immunochemical method for citrinin. Methods Mol. Biol., 157, 195-204.

Albers, G.W., Clark, W.M., Atkinson, R.P., Madden, K., Data, J.L. and Whitehouse, M.J. (1999): Dose escalation study of the NMDA glycine-site antagonist licostinel in acute ischemic stroke. Stroke, 30, 508-513.

Aleo, M.D., Wyatt, R.D. and Schnellmann, R.G. (1991): The role of altered mitochondrial function in citrinin-induced toxicity to rat renal proximal tubule suspensions. Toxicol. Appl. Pharmacol., 109, 455-463.

Ambrose, A.M. and DeEds, F. (1946): Some toxicological and pharmacological properties of citrinin. J. Pharmacol. Exp. Ther., 88, 173-186.

Arias, R.L., Tasse, J.R. and Bowlby, M.R. (1999): Neuroprotective interaction effects of NMDA and AMPA receptor antagonists in an in vitro model of cerebral ischemia. Brain Res., 816, 299308.

Berndt, W.O., Hayes, A.W. and Baggett, J.M. (1984): Effects of fungal toxins on renal slice calcium balance. Toxicol. Appl. Pharmacol., 74, 78-85.

Blandini, F., Porter, R.H. and Greenamyre, J.T. (1996): Glutamate and Parkinson's disease. Mol. Neurobiol., 12, 73-94.

Bouslimi, A., Bouaziz, C., Ayed-Boussema, I., Hassen, W. and Bacha, H. (2008): Individual and combined effects of ochratoxin A and citrinin on viability and DNA fragmentation in cultured Vero cells and on chromosome aberrations in mice bone marrow cells. Toxicology, 251, 1-7.

Chagas, G.M., Campello, A.P. and Klüppel, M.L. (1992a): Mechanism of citrinin-induced dysfunction of mitochondria. I. Effects on respiration, enzyme activities and membrane potential of renal cortical mitochondria. J. Appl. Toxicol., 12, 123-129.

Chagas, G.M., Oliveira, B.M., Campello, A.P. and Klüppel, M.L. (1992b): Mechanism of citrinin-induced dysfunction of mitochondria. II. Effect on respiration, enzyme activities, and membrane potential of liver mitochondria. Cell Biochem. Funct., 10, 209-216.

Chan, W.H. (2008): Citrinin induces apoptosis in mouse embryonic stem cells. IUBMB Life, 60, 171-179.

Chen, C.C. and Chan, W.H. (2009): Inhibition of citrinin-induced apoptotic biochemical signaling in human hepatoma G2 cells by resveratrol. Int. J. Mol. Sci., 10, 3338-3357.

Choi, D.W. (1994): Calcium and excitotoxic neuronal injury. Ann.
N. Y. Acad. Sci., 747, 162-171.

Da Lozzo, E.J., Oliveira, M.B. and Carnieri, E.G. (1998): Citrinininduced mitochondrial permeability transition. J. Biochem. Mol. Toxicol., 12, 291-297.

Dirnagl, U., Iadecola, C. and Moskowitz, M.A. (1999): Pathobiology of ischaemic stroke: an integrated view. Trends Neurosci., 22, 391-397.

Dong, X.X., Wang, Y. and Qin, Z.H. (2009): Molecular mechanisms of excitotoxicity and their relevance to pathogenesis of neurodegenerative diseases. Acta Pharmacol. Sin., 30, 379-387.

Estrada Sánchez, A.M., Mejía-Toiber, J. and Massieu, L. (2008): Excitotoxic neuronal death and the pathogenesis of Huntington's disease. Arch. Med. Res., 39, 265-276.

Facci, L., Leon, A. and Skaper, S.D. (1990): Hypoglycemic neurotoxicity in vitro: involvement of excitatory amino acid receptors and attenuation by monosialoganglioside GM1. Neuroscience, 37, 709-716.

Flajs, D. and Peraica, M. (2009): Toxicological properties of citrinin. Arh. Hig. Rada Toksikol., 60, 457-464.

Friis, P., Hasselager, E. and Krogh, P. (1969): Isolation of citrinin and oxalic acid from Penicillium viridicatum Westling and their nephrotoxicity in rats and pigs. Acta Pathol. Microbiol. Scand., $77,559-560$.

Grotta, J., Clark, W., Coull, B., Pettigrew, L.C., Mackay, B., Goldstein, L.B., Meissner, I., Murphy, D. and LaRue, L. (1995): Safety and tolerability of the glutamate antagonist CGS 19755 (Selfotel) in patients with acute ischemic stroke. Results of a phase IIa randomized trial. Stroke, 26, 602-605.

Gupta, M., Dolui, A.K., Dey, S.N., Mukherjee, S., Majumder, U.K. and Batabyal, S.K. (1986): Effect of the mycotoxin citrinin on some hormones and on enzymes and substrates of the EmbdenMeyerhof pathway in mice. Toxicon, 24, 519-523.

Klarić, M.S., Zelježić, D., Rumora, L., Peraica, M., Pepeljnjak, S. and Domijan, A.M. (2012): A potential role of calcium in apoptosis and aberrant chromatin forms in porcine kidney PK15 cells induced by individual and combined ochratoxin $\mathrm{A}$ and citrinin. Arch. Toxicol., 86, 97-107.

Krogh, P., Hasselager, E. and Friis, P. (1970): Studies on fungal nephrotoxicity. 2. Isolation of two nephrotoxic compounds from Penicillium viridicatum Westling: citrinin and oxalic acid. Acta Pathol. Microbiol. Scand. B Microbiol. Immunol., 78, 401-413.

Krystal, J.H., Karper, L.P., Seibyl, J.P., Freeman, G.K., Delaney, R., Bremner, J.D., Heninger, G.R., Bowers, M.B.Jr. and Charney, D.S. (1994): Subanesthetic effects of the noncompetitive NMDA antagonist, ketamine, in humans. Psychotomimetic, perceptual, cognitive, and neuroendocrine responses. Arch. Gen. Psychiatry, 51, 199-214.

Kumar, R., Dwivedi, P.D., Dhawan, A., Das, M. and Ansari, K.M. (2011): Citrinin-generated reactive oxygen species cause cell cycle arrest leading to apoptosis via the intrinsic mitochondrial pathway in mouse skin. Toxicol. Sci., 122, 557-566.

Lau, A. and Tymianski, M. (2010): Glutamate receptors, neurotoxicity and neurodegeneration. Pflugers Arch., 460, 525-542.

Lee, S.M., Wong, M.D., Samii, A. and Hovda, D.A. (1999): Evidence for energy failure following irreversible traumatic brain injury. Ann. N. Y. Acad. Sci., 893, 337-340.

Li, Y., Li, X., Lee, U., Kang, J.S., Choi, H.D. and Sona, B.W. (2006): A new radical scavenging anthracene glycoside, asperflavin ribofuranoside, and polyketides from a marine isolate of the fungus microsporum. Chem. Pharm. Bull. (Tokyo), 54, 882883.

Liu, B.H., Wu, T.S., Su, M.C., Chung, C.P. and Yu, F.Y. (2005): 
Citrinin protect neurons against glutamate-induced excitotoxicity

Evaluation of citrinin occurrence and cytotoxicity in Monascus fermentation products. J. Agric. Food Chem., 53, 170-175.

Liu, B.H., Yu, F.Y., Wu, T.S., Li, S.Y., Su, M.C., Wang, M.C. and Shih, S.M. (2003): Evaluation of genotoxic risk and oxidative DNA damage in mammalian cells exposed to mycotoxins, patulin and citrinin. Toxicol. Appl. Pharmacol., 191, 255-263.

Löscher, W. and Hönack, D. (1992): The behavioural effects of MK-801 in rats: involvement of dopaminergic, serotonergic and noradrenergic systems. Eur. J. Pharmacol., 215, 199-208.

Máté, G., Gazdag, Z., Mike, N., Papp, G., Pócsi, I. and Pesti, M. (2014): Regulation of oxidative stress-induced cytotoxic processes of citrinin in the fission yeast Schizosaccharomyces pombe. Toxicon, 90, 155-166.

Michaels, R.L. and Rothman, S.M. (1990): Glutamate neurotoxicity in vitro: antagonist pharmacology and intracellular calcium concentrations. J. Neurosci., 10, 283-292.

Mount, C. and Downton, C. (2006): Alzheimer disease: progress or profit? Nat. Med., 12, 780-784.

Muir, K.W. and Lees, K.R. (1995): Clinical experience with excitatory amino acid antagonist drugs. Stroke, 26, 503-513.

Nelson, T.S., Kirby, L.K., Beasley, J.N., Johnson, Z.B. and Ciegler, A. (1985): The effect of drying method and storage time on citrinin activity in corn. Poult. Sci., 64, 464-468.

Olney, J.W., Collins, R.C. and Sloviter, R.S. (1986): Excitotoxic mechanisms of epileptic brain damage. Adv. Neurol., 44, 857877.

Pfeiffer, E., Gross, K. and Metzler, M. (1998): Aneuploidogenic and clastogenic potential of the mycotoxins citrinin and patulin. Carcinogenesis, 19, 1313-1318.

Sattler, R. and Tymianski, M. (2001): Molecular mechanisms of glutamate receptor-mediated excitotoxic neuronal cell death. Mol. Neurobiol., 24, 107-129.

Schneider, L.S., Dagerman, K.S., Higgins, J.P. and McShane, R. (2011): Lack of evidence for the efficacy of memantine in mild Alzheimer disease. Arch. Neurol., 68, 991-998.

Schubert, D. and Piasecki, D. (2001): Oxidative glutamate toxicity can be a component of the excitotoxicity cascade. J. Neurosci., 21, 7455-7462.

Scott, P.M., Van Walbeek, W., Kennedy, B. and Anyeti, D. (1972): Mycotoxins (ochratoxin A, citrinin, and sterigmatocystin) and toxigenic fungi in grains and other agricultural products. J.
Agric. Food Chem., 20, 1103-1109.

Shaw, C.E., al-Chalabi, A. and Leigh, N. (2001): Progress in the pathogenesis of amyotrophic lateral sclerosis. Curr. Neurol. Neurosci. Rep., 1, 69-76.

Shinoda, Y., Ahmed, S., Ramachandran, B., Bharat, V., Brockelt, D., Altas, B. and Dean, C. (2014): BDNF enhances spontaneous and activity-dependent neurotransmitter release at excitatory terminals but not at inhibitory terminals in hippocampal neurons. Front. Synaptic Neurosci., 6, 27.

Shinoda, Y., Sadakata, T., Nakao, K., Katoh-Semba, R., Kinameri, E., Furuya, A., Yanagawa, Y., Hirase, H. and Furuichi, T. (2011): Calcium-dependent activator protein for secretion 2 (CAPS2) promotes BDNF secretion and is critical for the development of GABAergic interneuron network. Proc. Natl. Acad. Sci. USA, 108, 373-378.

Shinoda, Y., Tanaka, T., Tominaga-Yoshino, K. and Ogura, A. (2010): Persistent synapse loss induced by repetitive LTD in developing rat hippocampal neurons. PLoS One, 5, e10390.

Szydlowska, K. and Tymianski, M. (2010): Calcium, ischemia and excitotoxicity. Cell Calcium, 47, 122-129.

Tricklebank, M.D., Singh, L., Oles, R.J., Preston, C. and Iversen, S.D. (1989): The behavioural effects of MK-801: a comparison with antagonists acting non-competitively and competitively at the NMDA receptor. Eur. J. Pharmacol., 167, 127-135.

Van Den Bosch, L., Van Damme, P., Bogaert, E. and Robberecht, W. (2006): The role of excitotoxicity in the pathogenesis of amyotrophic lateral sclerosis. Biochim. Biophys. Acta, 1762, 10681082.

Villoslada, P., Arrondo, G., Sepulcre, J., Alegre, M. and Artieda, J. (2009): Memantine induces reversible neurologic impairment in patients with MS. Neurology, 72, 1630-1633.

Wang, Y. and Qin, Z.H. (2010): Molecular and cellular mechanisms of excitotoxic neuronal death. Apoptosis, 15, 1382-1402.

Yu, F.Y., Liao, Y.C., Chang, C.H. and Liu, B.H. (2006): Citrinin induces apoptosis in HL-60 cells via activation of the mitochondrial pathway. Toxicol. Lett., 161, 143-151.

Zhang, D., Li, X., Kang, J.S., Choi, H.D., Jung, J.H. and Son, B.W. (2007): Redoxcitrinin, a biogenetic precursor of citrinin from marine isolate of fungus Penicillium sp. J. Microbiol. Biotechnol., 17, 865-867. 